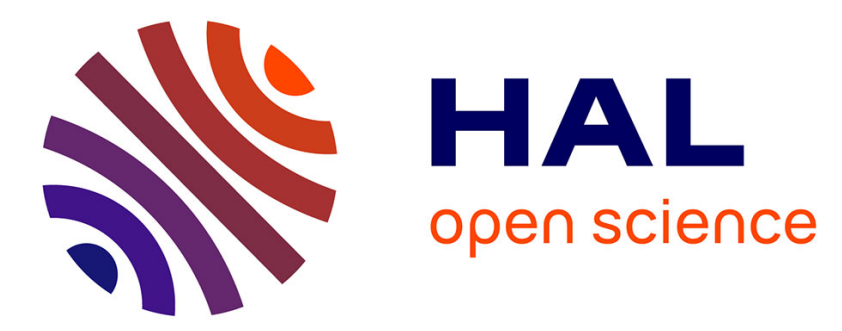

\title{
Que changent les nouvelles recommandations françaises du traitement de l'ostéoporose post-ménopausique ?
}

H. Blain

\section{To cite this version:}

H. Blain. Que changent les nouvelles recommandations françaises du traitement de l'ostéoporose post-ménopausique ?. La Revue de Médecine Interne, 2019, 40 (5), pp.275-277. 10.1016/j.revmed.2018.05.008 . hal-02549945

\section{HAL Id: hal-02549945 \\ https://hal.umontpellier.fr/hal-02549945}

Submitted on 22 Oct 2021

HAL is a multi-disciplinary open access archive for the deposit and dissemination of scientific research documents, whether they are published or not. The documents may come from teaching and research institutions in France or abroad, or from public or private research centers.
L'archive ouverte pluridisciplinaire HAL, est destinée au dépôt et à la diffusion de documents scientifiques de niveau recherche, publiés ou non, émanant des établissements d'enseignement et de recherche français ou étrangers, des laboratoires publics ou privés.

\section{다)(1) $(5$}

Distributed under a Creative Commons Attribution - NonCommerciall 4.0 International 
Version of Record: https://www.sciencedirect.com/science/article/pii/S0248866318305794

Manuscript_85b4ed36f81f1301ddbcf0b4c5dda1e0

\section{Editorial}

\section{Que changent les nouvelles recommandations françaises du traitement de l'ostéoporose post-ménopausique ?}

Mots clé : ostéoporose ; fracture ; recommandations Key words : osteoporosis ; fracture ; guidelines

Professeur Hubert BLAIN

Service de médecine Interne-Gériatrie

Pôle de gérontologie du CHU de Montpellier. Université de Montpellier.

Centre Antonin Balmes, 39 avenue Charles Flahaut, 34295 Montpellier Cedex 5

h-blain@chu-montpellier.fr Tél :0467336778 Fax : 0467330948 


\title{
Editorial
}

\section{Que changent les nouvelles recommandations françaises du traitement de l'ostéoporose post-ménopausique?}

\author{
Mots clé : ostéoporose ; fracture ; recommandations \\ Key words : osteoporosis ; fracture ; guidelines
}

Les nouvelles recommandations françaises du traitement de l'ostéoporose postménopausique viennent d'être publiées par le Groupement de Recherche et d'Information sur les Ostéoporoses (GRIO) en collaboration avec différentes Sociétés de spécialités médicales et chirurgicales [1]. Ces nouvelles recommandations répondent à un besoin de simplification des recommandations de 2012. Elles tiennent compte de nouvelles données sur l'épidémiologie et les facteurs de risque de fractures, en particulier imminentes, et de nouveaux traitements de l'ostéoporose.

Si nous assistons en France à une diminution de l'incidence relative des fractures de hanche chez les femmes de 40 ans et plus, l'augmentation en parallèle du nombre de femmes dans cette tranche d'âge explique au total une augmentation du nombre absolu de fractures de hanche de $5 \%$ chez les femmes ménopausées dans la dernière décade. Ces données sont de même ordre pour l'ensemble des autres sites fracturaires. Ainsi, même si nous n'observons pas l'augmentation exponentielle du nombre de fractures redoutée il y a quelques années, l'ostéoporose fracturaire reste un problème de santé publique majeur en deuxième période de vie chez la femme.

En dehors du risque de chute, le caractère récent d'une fracture ostéoporotique ou de fragilité (complications d'un traumatisme de basse énergie, c'est-à-dire dont l'intensité est inférieure ou égale à une chute de sa hauteur) représente le premier facteur de risque de fracture à court terme. Par exemple, après une fracture vertébrale, $25 \%$ des femmes présentent une nouvelle fracture vertébrale dans l'année qui suit. Or, contrairement aux autres fractures, les fractures vertébrales peuvent être pauci-symptomatiques et dès lors non diagnostiquées, risquant d'engager la patiente vers une cascade fracturaire en l'absence de traitement adapté. Une radiographie de rachis ou une évaluation densitométrique avec VFA (Vertebral Fracture Assessment) se justifie dès lors à la recherche d'une fracture vertébrale chez la femme ménopausée en cas de rachialgie, de diminution de taille d'au moins $4 \mathrm{~cm}$ au moins par rapport à la taille à 20 ans, en cas de perte de taille d'au moins $2 \mathrm{~cm}$ entre deux mesures et en présence de maladies chroniques et de traitements inducteurs de fractures vertébrales, dont les anti-aromatases et la corticothérapie. Cette recommandation de mesure régulière de taille et la prise d'informations sur les rachialgies pour diagnostiquer des fractures vertébrales pauci-symptomatiques intéresse tout particulièrement les médecins internistes qui traitent des pathologies chroniques, inflammatoires en particulier, et utilisent des corticoïdes (ISCD 2015) (www.iscd.org).

Le risque de fracture est inversement proportionnel à la densité minérale osseuse (DMO), au mieux analysée au col du fémur, la DMO du rachis pouvant être surestimée par l'arthrose ou les calcifications vasculaires en particulier. Les nouvelles recommandations insistent sur le fait qu'une ostéodensitométrie doit être systématiquement réalisée pour évaluer l'indication d'un traitement anti-ostéoporotique et, en cas de fracture, pour évoquer un autre diagnostic qu'une ostéoporose quand le T-score est supérieur à -1. La mesure de la DMO sera en outre utile pour décider de la poursuite du traitement après chaque cycle de 3 à 5 ans de traitement.

En dehors de la fracture, l'ostéodensitométrie est indiquée dans de nombreuses situations fréquemment rencontrées en médecine interne chez les femmes ménopausées: risque significatif de chute (antécédent de chute dans la dernière année; troubles de la marche évalué par exemple par un timed up and go $>14$ secondes; trouble de l'équilibre évalué par exemple par un temps d'appui unipodal non tenu 5 secondes; réduction de force musculaire, évaluée par exemple par un relever d'une chaise sans les mains non réalisé en 12 secondes; ou tout simplement une peur de tomber ou une marche précautionneuse)[2], corticothérapie de plus de 3 mois à une posologie $\geq 7,5 \mathrm{mg}$ 
équivalent prednisone, hypogonadisme prolongé, endocrinopathies telles que l'hypercorticisme, l'hyperparathyroïdie ou l'hyperthyroïdie, l'ostéogénèse imparfaite, antécédent familial au premier degré de fracture de l'extrémité supérieure du fémur ou en cas de dénutrition (indice de masse corporelle < 19).

Les nouvelles recommandations simplifient la prise de décision thérapeutique puisqu'elles indiquent un traitement anti-ostéoporotique:

- En cas de T-score $\leq-1$ après une fracture sévère (extrémité supérieure et inférieure du fémur, extrémité proximale du tibia et de l'humérus, bassin, vertèbre). Un t-score > 1 évoque une autre cause de fragilité osseuse que l'ostéoporose. Ces fractures sont dites sévères car elles témoignent d'une fragilité osseuse importante, exposant à un risque élevé de récidive fracturaire et à un pronostic fonctionnel et vital péjoratif en l'absence de traitement anti-ostéoporotique, de prévention des chutes chez les personnes à risque, et de traitement des comorbidités associées.

- En cas de T-score $\leq-2$ après une fracture non sévère touchant un site pouvant être atteint par l'ostéoporose. La main, le pied, les premières vertèbres dorsales et cervicales et le crâne ne sont pas des sites de la maladie ostéoporotique et une fracture sur ces sites évoque d'autres diagnostics que l'ostéoporose.

- $\quad$ En cas de T-score $\leq-3$, en l'absence de fracture.

Dans ces trois situations à haut risque de fracture ou de re-fracture, le nombre de sujets à traiter par un traitement anti-ostéoporotique pour prévenir une fracture justifie sauf contre-indication l'instauration de ce type de traitement. Par exemple le nombre de sujets à traiter (NNT) par le denosumab pour prévenir une fracture vertébrale morphométrique à 3 ans est de 15 chez les femmes de 75 ans et plus ayant un t-score fémoral bas, de 11 en cas d'antécédent de fracture vertébrale et de 9 quand les femmes ont les deux facteurs de risque (DMO basse et antécédent de fracture vertébrale)[3]. Dans cette même étude, le nombre de femmes à traiter pour prévenir une fracture de hanche est de 42 chez les femmes de 75 ans ou plus ayant un t-score bas [3]. Aucune étude comparative d'efficacité n'a été réalisée entre les molécules anti-ostéoporotiques, dont les études pivotales ont été réalisées chez des femmes ménopausées à haut risque de fracture [ostéoporose accompagnée ou non de fracture(s)] mais dont le risque de fracture était cependant variable (antécédents ou non de fracture(s), âge et DMO moyenne différents entre les études expliquant des incidences fracturaires dans les groupes contrôles variables entre les études). Si ces différences rendent difficiles la comparaison d'efficacité, les traitements injectables sont accompagnés d'une réduction relative de fractures plus élevée que les traitements oraux. Globalement, bisphosphonates et denosumab ont montré leur efficience à prévenir les fractures vertébrales et non vertébrales, en particulier de hanche, et le raloxifène et le tériparatide, à réduire l'incidence des fractures vertébrales. La plus grande efficacité des traitements anti-ostéoporotiques sur l'incidence des fractures vertébrales que non vertébrales est expliquée par le fait que les fractures vertébrales sont essentiellement liées à une fragilité osseuse alors que les fractures non vertébrales, en particulier de hanche, dépendent aussi du risque de chute. Dès lors des mesures pour prévenir les chutes devront être associées systématiquement au traitement antiostéoporotique chez les sujets à risque, c'est à dire ayant au moins un des facteurs suivants: antécédent de chute; troubles de l'équilibre ; trouble de la marche; faible force musculaire ; peur de tomber ; marche précautionneuse [2].

Les nouvelles recommandations confirment la nécessité, avant de débuter un traitement anti-ostéoporotique, de réaliser un bilan biologique pour confirmer le caractère primaire de l'ostéoporose et éliminer une contre-indication aux traitements anti-ostéoporotiques (bilan phosphocalcique, inflammatoire, clairance de la créatininémie, TSH, 25OH vitamine D, électrophorèse des protéines, bilan hépatique, numération formule sanguine) et de normaliser le statut vitamino-calcique.

Les nouvelles recommandations confirment que les biphosphonates oraux doivent être utilisés en première intention dans le traitement de l'ostéoporose post-ménopausique en l'absence de contre-indication (clairance de la créatinine inférieure à $30 \mathrm{ml} / \mathrm{min}$, hypocalcémie). Elle 
recommandent pour la première fois l'utilisation en première intention des traitements antiostéoporotiques injectables en cas de fracture sévère, de DMO très basse, de comorbidités (troubles mnésiques notamment), ou de défaut d'observance, en particulier dû à la polymédication, situations très fréquentes en médecine interne. Après une fracture de l'extrémité supérieure du fémur sera proposée en première intention une injection annuelle d'acide zolédronique, seul traitement à avoir montré une réduction de mortalité chez ces patients $(9,6 \%$ vs $13,3 \%$ à 1,9 ans en moyenne; $p<0,01)$ [4]. En présence d'au moins deux fractures vertébrales sera proposé en première intention le tériparatide en sous-cutanée quotidienne, en l'absence de contre-indication, incluant l'hypercalcémie et l'hyper-remodelage osseux, tumoral ou non. En effet, ce traitement réduit à 24 mois davantage que les biphosphonates oraux le risque de nouvelle fracture vertébrale morphométrique et clinique en cas d'ostéoporose fracturaire vertébrale $(5,4 \%$ vs $12,0 \% ; p<0,0001$ et $4,8 \%$ vs $9,8 \% ; p=0,0009$, respectivement) [5]. Ce traitement ostéoformateur administré pendant 18 à 24 mois (il est remboursé pendant 18 mois) sera relayé par un traitement anti-résorbeur, biphosphonate ou denosumab. Le denosumab, utilisé en injection sous-cutanée semestrielle, est indiqué en seconde intention, en relais des biphosphonates ou en cas de contre-indication à ceux-ci. Il est comme les biphosphonates contre indiqué en cas d'hypocalcémie. Des fractures vertébrales ont été décrites à l'arrêt du denosumab, suggérant l'intérêt d'un relais par biphosphonates pendant 6 à 12 mois à l'arrêt de ce traitement. Biphosphonates et denosumab peuvent être associés au développement très rare d'une ostéonécrose de la mâchoire, indiquant de prévoir les soins dentaires et en particulier les avulsions dentaires si possible avant le démarrage de ces traitements sauf en cas de risque élevé de fracture à court terme (cas de fracture sévère en particulier) où le traitement antiostéoporotique ne doit pas être retardé (l'efficacité anti-fracturaire des traitement débute à 6 mois et le plein effet est observé après 12 mois). Biphosphonates et denosumab peuvent aussi être associés au développement très rare de fractures atypiques du fémur (fractures en bâton de craie, c'est-à-dire non comminutive, avec un trait de fracture perpendiculaire à la corticale), nécessitant l'arrêt de ces traitements dès le diagnostic posé. Le raloxifène est à réserver aux patientes de moins de 70 ans avec un risque de fracture périphérique faible (faible risque de chute, absence de fracture périphérique, T-score au col du fémur > -3). Le traitement hormonal de la ménopause (THM) pourra être utilisé entre 50 et 60 ans en cas de signes climatériques de la ménopause ou en cas d'intolérance ou d'inefficacité des autres traitements. Si le THM est utilisé à une dose plus faible que recommandée pour une protection osseuse efficace, une ostéodensitométrie doit être répétée après 2 ou 3 ans de traitement et un traitement anti-ostéoporotique peut être ajouté au THM si la DMO reste basse.

Un avis spécialisé est requis en cas de fracture sévère quand le T-score est supérieur à -1, de fracture non sévère quand le T-Score est compris entre -2 et -1 , et en l'absence de fracture quand le T-Score est compris entre -3 et -2 . Lors de cet avis spécialisé la décision thérapeutique pourra être aidée par les scores de risque de fracture comme le $F R A X^{\circledR}$ et l'utilisation de seuils d'indication thérapeutique dépendant de l'âge (www.sheffield.ac.uk/FRAX). Dans les autres cas (absence d'indication de traitement ou d'avis spécialisé), un traitement anti-ostéoporotique n'est pas recommandé.

Dans les nouvelles recommandations, le dosage du CTX sérique (dosé à jeun, le matin, au moins 6 mois après une fracture), marqueur de la résorption osseuse, peut-être réalisé entre le troisième et le douzième mois d'un traitement anti-résorbeur (biphosphonates, denosumab, raloxifène, THM) en cas de doute sur l'observance ou pour évaluer l'efficacité du traitement (en cas de risque de refracture important, en particulier après fracture sévère). Quand le CTX reste supérieur aux valeurs normales des femmes non ménopausées, signe que le traitement est insuffisant pour réduire significativement le remodelage osseux, une réévaluation de l'observance est nécessaire, et, en cas d'observance satisfaisante, un changement de traitement peut-être proposé. II s'agit d'une différence avec les recommandations de la Haute Autorité de Santé qui ne recommandent pas l'utilisation des marqueurs de remodelage dans le suivi des patientes recevant un traitement antiostéoporotiques. 
Après 3 à 5 ans, l'arrêt du traitement peut être discuté en l'absence de nouvelle fracture ou de nouveau facteur de risque de fracture, en l'absence de baisse de DMO supérieures à $0,03 \mathrm{~g} / \mathrm{cm}^{2}$ au rachis ou à la hanche et, après une fracture sévère, quand le T-score n'est pas inférieur à -2 ou 2,5 . Une réévaluation est recommandée après 2 ans d'arrêt de traitement, la rémanence d'effet osseux de l'acide zolédronique et de l'alendronate étant plus longue que celle du risédronate, le denosumab n'ayant pas de rémanence d'effet à son arrêt.

Toutes les études montrent que l'ostéodensitométrie est peu réalisée chez les patientes à risque d'ostéoporose, en particulier après fracture, que les traitements anti-ostéoporotiques ne sont pas suffisamment utilisés chez les femmes à haut risque de fracture et que l'observance de ces traitements est faible, en particulier après une fracture $(15 \%$ des femmes ont eu une ostéodensitométrie et $15 \%$ reçoivent un traitement un an après la fracture). Pour toutes ces raisons, les nouvelles recommandations proposent la création de filières "fracture-orthogériatriques", permettant une évaluation multi-disciplinaire des patients fracturaires, incluant un avis rhumatologique et un avis gériatrique/de médecine interne en cas de risque de chute ou de comorbidités, de manière à instaurer et effectuer le suivi des traitements anti-ostéoporotiques et mettre en place et organiser le suivi des mesures visant à prévenir les chutes et équilibrer les comorbidités [2]. Une méta-analyse des études menées sur l'efficacité des "fracture liaison services" (appellation anglophone qui pourrait se traduire par "service de liaison des fractures) a montré qu'après une fracture, la prise en charge dans ces structures permet d'augmenter le nombre de patients chez qui est réalisée une ostéodensitométrie, le nombre de traitements antiostéoporotiques initiés et l'adhérence au traitement d'une part, et de réduire le risque de re-fracture et la mortalité d'autre part. Le nombre de personnes à prendre en charge dans une filière " fractureorthogériatrique " pour augmenter de 1 le nombre d'ostéodensitométries réalisées, de traitements anti-ostéoporotiques initiés, et de patients observants pour ces traitements est de 4,5 et 4 , respectivement. Le nombre de personnes à prendre en charge dans une filière "fractureorthogériatrique » pour prévenir une fracture est de 20, il est de 33 pour prévenir un décès [6].

\section{Conflits d'intérêt :}

Pas de conflit d'intérêt.

\section{Références :}

1. Briot K, Roux C, Thomas T, Blain H, Buchon D, Chapurlat R, et al. 2018 Update of French Recommendations on the Management of Postmenopausal Osteoporosis. Joint Bone Spine. 2018 pii: S1297-319X(18)30062-9.

2. Blain H, Masud T, Dargent-Molina P, Martin FC, Rosendahl E, van der Velde N, et al. EUGMS Falls and Fracture Interest Group; European Society for Clinical and Economic Aspects of Osteoporosis and Osteoarthritis (ESCEO), Osteoporosis Research and Information Group (GRIO), and International osteoporosis Foundation (IOF). A Comprehensive Fracture Prevention Strategy in Older Adults: The European Union Geriatric Medicine Society (EUGMS) Statement. J Nutr Health Aging. 2016;20:64752.

3. Boonen S, Adachi JD, Man Z, Cummings SR, Lippuner K, Törring O, et al. Treatment with denosumab reduces the incidence of new vertebral and hip fractures in postmenopausal women at high risk. J Clin Endocrinol Metab. 201196:1727-36.

4. Lyles KW, Colón-Emeric CS, Magaziner JS, Adachi JD, Pieper CF, Mautalen C, et al. HORIZON Recurrent Fracture Trial. Zoledronic acid and clinical fractures and mortality after hip fracture. N Engl J Med. 2007;357:1799-809. 
5. Kendler DL, Marin F, Zerbini CAF, Russo LA, Greenspan SL, Zikan V, et al. Effects of teriparatide and risedronate on new fractures in post-menopausal women with severe osteoporosis (VERO): a multicentre, double-blind, double-dummy, randomised controlled trial. Lancet. 2018;391:230-40.

6. Wu CH, Tu ST, Chang YF, Chan DC, Chien JT, Lin $\mathrm{CH}$, et al. Fracture liaison services improve outcomes of patients with osteoporosis-related fractures: A systematic literature review and metaanalysis. Bone. 2018;111:92-100. 